\title{
Magnitud de efecto de un programa dirigido a cuidadores familiares de personas con enfermedad crónica ${ }^{1}$

\author{
Effect magnitude of a program for family caregivers of people with \\ chronic disease
}

Título abreviado: Efecto de un programa para cuidadores de enfermos crónicos

Sonia Carreño-Moreno², Lorena Chaparro-Díaz ${ }^{3}$, Lucenith Criado ${ }^{4}$, Olga Marina Vega ${ }^{5}$, Ivonne Cuenca ${ }^{6}$

\section{Resumen}

Objetivo. Evaluar el efecto de programa "Cuidando a los Cuidadores" en la habilidad de cuidado y sobrecarga en cuidadores familiares y determinar la magnitud del efecto. Método. Estudio cuantitativo con abordaje preexperimental con un grupo de intervención de 329 cuidadores. Los instrumentos fueron el Inventario de Habilidad de Cuidado (CAI) y entrevista de sobrecarga de Zarit. Se analizaron los datos con la prueba $\mathrm{T}$ de Student y el efecto fue calculado con la d de Cohen y coeficiente r. Resultados. Se encontró aumentó significativo en la habilidad de cuidado y efecto fue moderado. La sobrecarga disminuyó después de la intervención y el efecto fue moderado especialmente para la dimensión interpersonal.

Palabras claves: enfermedad crónica, cuidadores, aptitud, efectividad, costo de la enfermedad.

\section{Abstract}

Objective. Evaluate the effect to Programa "Cuidando a los Cuidadores" and determine the magnitude of the effect. Method. Quantitative study with pre-experimental approach with 329 family caregivers. The measurers was Caring Ability Inventory (CAI) and Zarit Burden Interview. The data was analyzed with Student's t test and the effect was calculated using Cohen's $\mathrm{d}$ and coefficient $r$. Results. The caring ability increased significantly and the effect was moderate. The burden decreased after intervention and the effect was moderate, especially for interpersonal dimension.

Keywords: Chronic illness; Family Caregivers; aptitude; Cost of Illness.

\footnotetext{
1. Trabajo enmarcado en el Programa para la disminución de la carga de la Enfermedad Crónica No Transmisible en Colombia, Financiado por Colciencias. Contrato 366-2011, código del programa 501753730890, Codigo Hermes 16875.

2. Profesora Asistente, Universidad Nacional de Colombia (Sede Bogotá), Facultad de Enfermería, Departamento de Enfermería- Grupo de Investigación Cuidado de Enfermería al paciente crónico. Carrera 30 No. 45-03. Bogotá, Colombia. Teléfono: +5713165000 , extensión: 10340. Correspondencia: spcarrenom@unal.edu.co

3. Enfermera, Doctora en Enfermería, Profesora asociada, Universidad Nacional de Colombia (Sede Bogotá), Facultad de Enfermería- Grupo de Investigación Cuidado de Enfermería al paciente crónico. Carrera 30 No.45-03, Edif. 228 of. 301. Bogotá, Código Postal: 111321,

Colombia. Correspondencia: olchaparrod@unal.edu.co

4. Profesora Asistente, Fundación Universitaria de San Gil Unisangil- Facultad de Ciencias de la Educación y de la Salud-Grupo de

Investigación Ciencias de la Educación y de la Salud Ices. Correspondencia: mcriado@unisangil.edu.co

5. Profesora titular, Universidad Francisco de Paula Santander (Cúcuta), Programa de Enfermería, Grupo de Investigación de Cuidado de

Enfermería. Correspondencia: olgavega@ufps.edu.co

6. Enfermera especialista. Clínicas renales RTS SAS. Bogotá. Colombia. Correspondencia: ivonne_gloria_cuenca@baxter.com
} 


\section{Introducción}

La Enfermedad Crónica No Transmisible (ECNT) tiene un significado particular para quien vive la experiencia por lo que representan la sintomatología y el impacto en la calidad de vida; sin embargo, es muy poco el reconocimiento a quien asume el cuidado (cuidador familiar), por parte de la misma familia, los sistemas de salud o los profesionales involucrados en la atención $(1,2)$.

$\mathrm{Al}$ respecto, se conoce que la vivencia del cuidador familiar representa una sobrecarga importante a nivel físico, emocional, económico y social; sus formas de expresión por lo general son la depresión, la ansiedad y el estrés relacionado con el cumplimiento de actividades instrumentales de la vida diaria y a largo plazo la insatisfacción con la labor y, en muchos, la aparición del síndrome del cuidador y el desenlace con la aparición de enfermedades crónicas o incluso la muerte (3).

En este sentido, en Colombia se ha indagado el concepto de habilidad de cuidado en los cuidadores familiares (4) y es un lineamiento que guía intervenciones desde enfermería (5), pero que es útil reconocerlo para otras disciplinas asociadas al cuidado informal.

El programa "Cuidando a los Cuidadores" fue creado como respuesta a la problemática de la vivencia de cuidar una persona en situación de enfermedad crónica en Colombia y Latinoamérica, con el fin de fortalecer la habilidad de cuidado de los cuidadores familiares de las personas en situación de cronicidad (6). Se realizó bajo los tres lineamientos conceptuales de Ngozy Nkongo: 1) conocimiento, que implica saber cuáles son las necesidades, fortalezas y debilidades de la persona que se cuida; 2) valor, que se obtiene con la experiencia del pasado para estar abierto a las necesidades del presente, implica tomar decisiones y buscar soporte, en otros; y 3) paciencia, que comprende la búsqueda de crecimiento y significado a través de la exploración, expresión, reflexión y tolerancia ante la desorganización (7). El programa está disponible en dos versiones; la original, que se compone de diez sesiones, una sesión de inducción y tres por cada dimensión; y la versión institucional, que consta de cuatro sesiones, una de inducción y una sesión por cada dimensión.

En la versión original, el programa ha sido sometido a prueba en varios estudios con resultados variables. Para el caso de los cuidadores de niños con discapacidad (8), encontraron que el programa no tiene efecto en la habilidad de cuidado, aunque a nivel descriptivo hay un leve aumento de las dimensiones conocimiento y paciencia. En cuidadores familiares de personas con enfermedad crónica (9-11) reportaron efecto del programa en el aumento de las dimensiones conocimiento, paciencia y en habilidad de cuidado total $(\mathrm{p}<0.05)$.

En la versión institucional se reportó un efecto del programa en el aumento de las dimensiones conocimiento, paciencia y en la habilidad de cuidado total $(\mathrm{p}<0.001)$, en cuidadores familiares de niños con cáncer (5).

En el marco del programa para la disminución de la enfermedad crónica no trasmisible (ECNT) en Colombia, se ha propuesto el modelo para abordar la carga del cuidado de la ECNT; el cual, dentro de su estructura interna, muestra una relación inversa entre la habilidad de cuidado y la sobrecarga del cuidador (12). Con el argumento anterior y considerando que el programa "Cuidando a los Cuidadores" en su versión institucional ha sido sometido a prueba sólo en cuidadores familiares de niños con cáncer y su efecto sólo ha sido evaluado en la habilidad de cuidado; se buscó evaluar si hay efecto del programa en la habilidad de cuidado y sobrecarga en cuidadores familiares de enfermos crónicos y cuál era la magnitud del mismo.

\section{Materiales y métodos}

Estudio cuantitativo de abordaje preexperimental, con un solo grupo de intervención y mediciones 
pre y pos prueba $(13,14)$. Fueron incluidos en el estudio 329 cuidadores familiares de personas con Enfermedad Crónica No Transmisible (ECNT), que cumplían con los siguientes criterios: tener 18 años o más, llevar seis o más meses como cuidador, y tener un estado mental intacto. Las variables medidas fueron: en la preprueba, variables de caracterización de la diada (persona con ECNT y cuidador familiar), habilidad de cuidado y sobrecarga del cuidador; los dos últimos fueron medidos nuevamente en la posprueba del estudio en el momento en que finalizó la intervención. Los instrumentos utilizados fueron: encuesta de caracterización de la diada (15) la cual tiene validez aparente, Validez facial y validez de expertos. Para medir la habilidad de cuidado se usó el Inventario de Habilidad de Cuidado
(CAI) creado por Nkongo (7), instrumento de 37 ítems con respuesta tipo likert de 1 a 7 , en donde 1 equivale a totalmente en desacuerdo y 7 totalmente de acuerdo; el instrumento tiene un Alfa de Cronbach de 0,89 y estabilidad de $r=0,80$ en Colombia (16). Para medir la sobrecarga del cuidador se usó la Entrevista Zarit, instrumento de 22 ítems con respuesta Likert de 1 a 5, en donde 1 es nunca y 5 es siempre; tiene validez de constructo y alfa de Cronbach de 0,86 para Colombia (17).

La intervención, programa "Cuidando a los Cuidadores" versión institucional, consta de cuatro sesiones presenciales, grupales, con duración de dos horas cada una. Los módulos del programa se presentan en la tabla 1 .

Tabla 1. Programa "Cuidando a los Cuidadores".

\begin{tabular}{|c|c|c|}
\hline TEMAS & OBJETIVOS ESPECÍFICOS & ACTIVIDADES \\
\hline Inducción & $\begin{array}{l}\text { Establecer una relación empática } \\
\text { entre los participantes del } \\
\text { programa. }\end{array}$ & $\begin{array}{l}\text { Presentación personal, historia de vida y experiencia de } \\
\text { cuidado de una persona con ECNT. }\end{array}$ \\
\hline $\begin{array}{l}\text { Conocimiento } \\
\text { que se requiere } \\
\text { para comprender } \\
\text { y facilitar el rol } \\
\text { de cuidador } \\
\text { (Conocimiento }\end{array}$ & $\begin{array}{l}\text { Generar un espacio de } \\
\text { conocimiento y reconocimiento } \\
\text { de las personas implicadas en } \\
\text { el proceso de cuidado familiar } \\
\text { alrededor de la experiencia de } \\
\text { enfermedad crónica. }\end{array}$ & $\begin{array}{l}\text { Reconocer las cosas más lindas e importantes que tienen } \\
\text { las personas involucradas en el cuidado y la forma de } \\
\text { comunicarlo. } \\
\text { Aspectos que le cuestan trabajo y que le dan confianza al } \\
\text { cuidador. } \\
\text { Identificación de cuidados realizados consigo mismo y con el } \\
\text { otro. } \\
\text { Resolución de inquietudes con respecto al grado de } \\
\text { preparación para el cuidado. }\end{array}$ \\
\hline $\begin{array}{l}\text { La habilidad } \\
\text { social y la toma } \\
\text { de decisiones en } \\
\text { la tarea de cuidar } \\
\text { (Valor). }\end{array}$ & $\begin{array}{l}\text { Aplicar un modelo de decisiones } \\
\text { y reconocimiento de apoyos que } \\
\text { tiene el cuidador en su tarea de } \\
\text { cuidado. }\end{array}$ & $\begin{array}{l}\text { Las similitudes entre los cuidadores. } \\
\text { Afrontando las dificultades del cuidado. } \\
\text { Identificando el propio valor. } \\
\text { El proceso de toma de decisiones aplicado a las situaciones del } \\
\text { cuidado. } \\
\text { El fortalecimiento de la red de soporte social. } \\
\text { La previsión de apoyo y actuación en las emergencias. }\end{array}$ \\
\hline $\begin{array}{l}\text { La experiencia de } \\
\text { crecer y dimensionar } \\
\text { el significado del } \\
\text { cuidado (Paciencia). }\end{array}$ & $\begin{array}{l}\text { Redimensionar la experiencia de } \\
\text { ser cuidador. } \\
\text { Comprender el significado de } \\
\text { la paciencia como elemento de } \\
\text { crecimiento dentro del proceso de } \\
\text { cuidado. }\end{array}$ & $\begin{array}{l}\text { Lo que tranquiliza e impacienta al cuidador. } \\
\text { Identificación de qué pueden hacer los cuidadores para } \\
\text { trabajar la paciencia } \\
\text { Establecimiento de metas para incrementar el conocimiento, } \\
\text { el valor y la paciencia. } \\
\text { El cuidador hábil. } \\
\text { El cuidador con nuevas metas y estrategias. } \\
\text { Reconocerse a sí mismo como un cuidador competente que } \\
\text { requiere ayuda, orientación y descanso. } \\
\text { Lo que sucederá al futuro y cómo prepararse. }\end{array}$ \\
\hline
\end{tabular}

Fuente. Elaborado a partir de Grupo Cuidado de Enfermería al Paciente Crónico, 2012. 
Se trata de una intervención en la que los encuentros se basan en el compartir y reflexionar sobre experiencias vividas en la atención de una persona con ECNT en torno al conocimiento, el valor y la paciencia como elementos de la habilidad de cuidado. Los encuentros son guiados por un profesional de enfermería entrenado en el programa y se apoyan en una cartilla denominada Programa "Cuidando a los Cuidadores" (versión institucional) que contiene ejercicios de reflexión sobre los temas expuesto en la tabla 1.

Para el análisis de los datos se utilizó el programa SPSS versión 21 licenciado para la Universidad Nacional de Colombia. En las variables de caracterización se usó estadística descriptiva; para la comparación pre y pos prueba se aplicó la prueba de Kolgomorov Smirnov, generando un p valor de 0,0967 que indica no rechazar la hipótesis nula y por tanto aporta evidencia estadística para considerar que las variables de habilidad de cuidado y sobrecarga del cuidado se ajustan al modelo gaussiano. El estadístico de elección para la comparación pre y post en ambas variables es la prueba $\mathrm{T}$ de Student para muestras pareadas; para determinar el tamaño del efecto de la intervención se calculó con la d de Cohen que reporta el tamaño de efecto en desviaciones estándar; medida que posteriormente se expresó en términos de coeficiente $\mathrm{r}$, por ser un coeficiente comúnmente manejado e interpretado por la comunidad científica, pues aporta una idea de la intensidad del efecto, ya que entre más cercano este a \pm 1 , más fuerte es el efecto y, por ende, entre más cercano a cero es más débil.

Este trabajo cumplió con los principios éticos de justicia, reciprocidad, beneficencio y fue clasificado como de riesgo mínimo, se tuvo en cuenta la normatividad nacional (Ministerio de la protección social, 1993) y la específica para la disciplina de enfermería (18). El estudio contó con aval del Comité de Ética de la Facultad de Enfermería de la Universidad Nacional de Colombia (Aval: UGI- 3202013). Se aplicó el consentimiento informado a los participantes de cada uno de los grupos teniendo en cuenta también los principios de autonomía, justicia, veracidad y beneficencia.

Los participantes fueron captados en dos modalidades: invitación a grupos interesados (individual e institucional) e implementación institucional en diferentes regiones de Colombia. Se realizó en el marco del programa para la disminución de la Carga de la Enfermedad Crónica No transmisible en Colombia a través de nodos de la Red Latinoamericana de Cuidado al paciente crónico y la familia, y la Unión Temporal Carga de la Enfermedad Crónica No Transmisible en Colombia.

Para la recolección de los datos y aplicación del programa se garantizó capacitación previa y acompañamiento permanente para verificar la calidad del dato a través de videoconferencias, comunicaciones electrónicas y sesiones presenciales.

\section{Resultados}

\section{Características de los participantes}

En la tabla dos se presentan los hallazgos de caracterización de la persona con ECNT.

Tabla 2. Caracterización de la persona con ECNT.

\begin{tabular}{|l|l|l|}
\multicolumn{2}{|c|}{ VARIABLE } & $\begin{array}{c}\text { PORCENTAJE } \\
\%\end{array}$ \\
\hline \multirow{4}{*}{ GÉNERO } & Femenino & 41,9 \\
\cline { 2 - 3 } & Masculino & 58,1 \\
\hline \multirow{4}{*}{ EDAD } & Media & 43,2 \\
\cline { 2 - 3 } & Mediana & 44 \\
\cline { 2 - 3 } & Desviación estándar & 29,8 \\
\cline { 2 - 3 } & Mínimo & 2 \\
\cline { 2 - 3 } & Máximo & 99 \\
\hline \multirow{4}{*}{$\begin{array}{l}\text { TESTS SPMSQ (Status } \\
\text { mental- Número de } \\
\text { errores) }\end{array}$} & $6-8$ & 52,6 \\
\cline { 2 - 3 } & $9-11$ & 27,1 \\
\cline { 2 - 3 } & $12-24$ & 20,4 \\
\hline & $0-2$ & 70,5 \\
\cline { 2 - 3 } & $3-4$ & 9,4 \\
\cline { 2 - 3 } & $8-7$ & 3,6 \\
\hline
\end{tabular}




\begin{tabular}{|c|c|c|}
\hline \multirow{9}{*}{ ESCOLARIDAD } & Analfabeta & 11,9 \\
\hline & Primaria incompleta & 52 \\
\hline & Primaria completa & 15,2 \\
\hline & $\begin{array}{l}\text { Bachillerato } \\
\text { incompleto }\end{array}$ & 7,6 \\
\hline & Bachillerato completo & 4,6 \\
\hline & Técnico & 2,7 \\
\hline & $\begin{array}{l}\text { Universidad } \\
\text { incompleta }\end{array}$ & 0,3 \\
\hline & Universidad completa & 5,5 \\
\hline & Postgrados & 0,3 \\
\hline \multirow{5}{*}{ ESTADO CIVIL } & Casado(a) & 24,3 \\
\hline & Separado(a) & 5,2 \\
\hline & Soltero(a) & 53,5 \\
\hline & Unión libre & 4 \\
\hline & Viudo(a) & 13,1 \\
\hline \multirow{6}{*}{ OCUPACIÓN } & Empleado & 2,7 \\
\hline & Estudiante & 38,9 \\
\hline & Hogar & 40,1 \\
\hline & Pensionado & 11,6 \\
\hline & Trabajo independiente & 6,7 \\
\hline & Otra & 0 \\
\hline \multirow{4}{*}{$\begin{array}{l}\text { PERCEPCIÓN } \\
\text { SUBJETIVA DE SER } \\
\text { UNA CARGA PARA SU } \\
\text { FAMILIA }\end{array}$} & Muy Alta & 13,4 \\
\hline & Alta & 25,8 \\
\hline & Moderada & 27,4 \\
\hline & Baja & 33,4 \\
\hline
\end{tabular}

Fuente. Elaboración propia a partir de los datos de estudio de 2016.

En la tabla tres se presentan los hallazgos de caracterización del cuidador familiar.

Tabla 3. Caracterización del cuidador familiar.

\begin{tabular}{|l|l|l|}
\multicolumn{2}{|c|}{ VARIABLE } & $\begin{array}{c}\text { PORCENTAJE } \\
\%\end{array}$ \\
\hline \multirow{5}{*}{ GÉNERO } & Femenino & 89,7 \\
\cline { 2 - 3 } & Masculino & 10,3 \\
\hline \multirow{5}{*}{ EDAD } & Media & 46,9 \\
\cline { 2 - 3 } & Mediana & 47 \\
\cline { 2 - 3 } & Desviación estándar & 13,6 \\
\cline { 2 - 3 } & Mínimo & 18 \\
\cline { 2 - 3 } & Máximo & 77 \\
\hline \multirow{5}{*}{ ESCOLARIDAD } & Analfabeta & 1,2 \\
\cline { 2 - 3 } & Primaria incompleta & 17,9 \\
\cline { 2 - 3 } & Primaria completa & 18,8 \\
\hline
\end{tabular}

\begin{tabular}{|c|c|c|}
\hline & $\begin{array}{l}\text { Bachillerato } \\
\text { incompleto }\end{array}$ & 12,2 \\
\hline & Bachillerato completo & 24 \\
\hline & Técnico & 8,2 \\
\hline & $\begin{array}{l}\text { Universidad } \\
\text { incompleta }\end{array}$ & 5,2 \\
\hline & Universidad completa & 10 \\
\hline & Postgrados & 2,4 \\
\hline \multirow{5}{*}{ ESTADO CIVIL } & Casado(a) & 38 \\
\hline & Separado(a) & 9,7 \\
\hline & Soltero(a) & 26,4 \\
\hline & Unión libre & 21 \\
\hline & Viudo(a) & 4,9 \\
\hline \multirow{6}{*}{ OCUPACIÓN } & Empleado & 16,1 \\
\hline & Estudiante & 4 \\
\hline & Hogar & 50,8 \\
\hline & Pensionado & 0 \\
\hline & Trabajo independiente & 24,6 \\
\hline & Otra & 4,6 \\
\hline \multirow{2}{*}{ ÚNICO CUIDADOR } & $\mathrm{Si}$ & 61,1 \\
\hline & No & 38,9 \\
\hline \multirow{6}{*}{$\begin{array}{l}\text { PARENTESCO CON } \\
\text { LA PERSONA A QUIEN } \\
\text { CUIDA }\end{array}$} & Abuelo(a) & 0,9 \\
\hline & Amigo(a) & 2,1 \\
\hline & Esposo(a) & 13,7 \\
\hline & Hijo(a) & 25,5 \\
\hline & Madre o Padre & 49,2 \\
\hline & Otro & 8,5 \\
\hline
\end{tabular}

Fuente. Elaboración propia a partir de los datos de estudio de 2016.

En la tabla cuatro se presentan los hallazgos de caracterización que son comunes entre la díada persona con ECNT- cuidador familiar.

Tabla 4. Características comunes de la díada.

\begin{tabular}{|l|l|l|}
\hline \multicolumn{2}{|c|}{ VARIABLE } & $\begin{array}{c}\text { PORCENTAJE } \\
\%\end{array}$ \\
\hline \multirow{5}{*}{$\begin{array}{l}\text { ESTRATO } \\
\text { SOCIOECONÓMICO }\end{array}$} & 1 & 33,4 \\
\cline { 2 - 3 } & 2 & 33,7 \\
\cline { 2 - 3 } & 3 & 26,7 \\
\cline { 2 - 3 } & 4 & 4,3 \\
\cline { 2 - 3 } & 5 & 0,6 \\
\cline { 2 - 3 } & 6 & 1,2 \\
\hline
\end{tabular}




\begin{tabular}{|l|l|l|}
\hline \multirow{4}{*}{$\begin{array}{l}\text { TIEMPO EN } \\
\text { MESES EN LA }\end{array}$} & Media & 137,1 \\
\cline { 2 - 3 } $\begin{array}{l}\text { RELACIÓN DE } \\
\text { CUIDADO }\end{array}$ & $\begin{array}{l}\text { Mesiana } \\
\text { estándar }\end{array}$ & 108 \\
\cline { 2 - 3 } & Mínimo & 118,5 \\
\cline { 2 - 3 } & Máximo & 632 \\
\hline
\end{tabular}

\section{Evaluación efecto del programa "Cuidando a los Cuidadores”. Versión institucional}

En la tabla cinco se presentan los resultados de la prueba $\mathrm{T}$ de Student y el tamaño del efecto para la variable habilidad de cuidado y sus dimensiones.

Tabla 5. Comparación pretest-postest habilidad de cuidado y tamaño de efecto.

\begin{tabular}{|c|c|c|c|c|c|c|c|}
\hline \multicolumn{6}{|c|}{ EFECTO DEL PROGRAMA } & \multicolumn{2}{|c|}{$\begin{array}{l}\text { TAMAÑO DEL } \\
\text { EFECTO }\end{array}$} \\
\hline \multirow{2}{*}{ DIMENSIONES } & \multicolumn{2}{|c|}{$\begin{array}{l}\text { Estadísticos descriptivos de } \\
\text { la diferencia }\end{array}$} & \multirow{2}{*}{ T Student } & \multirow{2}{*}{$\begin{array}{l}\text { Grados } \\
\text { de } \\
\text { libertad }\end{array}$} & \multirow{2}{*}{$P$ valor } & \multirow{2}{*}{ d Cohen } & \multirow{2}{*}{$\mathrm{r}$} \\
\hline & Media & $\begin{array}{l}\text { Desviación } \\
\text { estándar }\end{array}$ & & & & & \\
\hline CONOCIMIENTO & $-11,30$ & 16,45 & $-12,419$ & 326 & $<0,01$ & $-0,95$ & $-0,4$ \\
\hline VALOR & $-9,52$ & 15,47 & $-11,134$ & 326 & $<0,01$ & $-0,81$ & $-0,37$ \\
\hline PACIENCIA & $-7,45$ & 11,44 & $-11,816$ & 328 & $<0,01$ & $-0,93$ & $-0,42$ \\
\hline TOTAL & $-28,55$ & 41,29 & $-12,466$ & 324 & $<0,01$ & $-0,96$ & $-0,43$ \\
\hline
\end{tabular}

Fuente. Elaboración propia a partir de los datos de estudio de 2016.

El gráfico 1 representa el cambio de la variable habi-

lidad de cuidado en la pre y pos prueba del estudio.

Gráfico 1. Habilidad de cuidado pre y pos prueba.

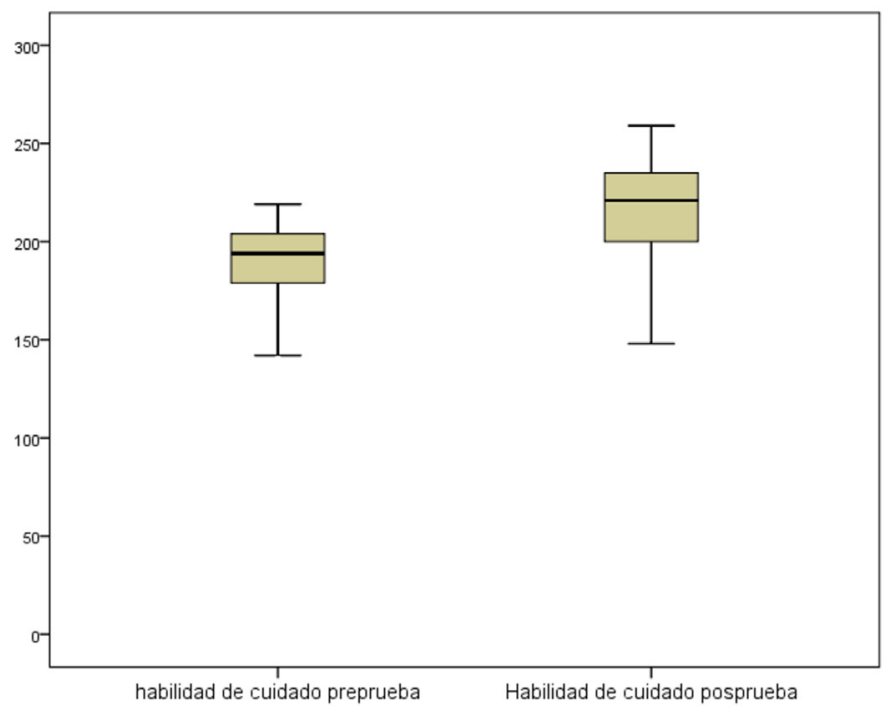

Fuente. Elaboración propia a partir de los datos de estudio de 2016. 
En la tabla seis se presentan los resultados de la prueba T de Student y tamaño del efecto para la variable sobrecarga del cuidador y sus dimensiones.

Tabla 6. Comparación pretest-postest sobrecarga del cuidador y tamaño de efecto.

\begin{tabular}{|c|c|c|c|c|c|c|c|}
\hline \multicolumn{6}{|c|}{ EFECTO DEL PROGRAMA } & \multicolumn{2}{|c|}{$\begin{array}{l}\text { TAMAÑO DEL } \\
\text { EFECTO }\end{array}$} \\
\hline \multirow{2}{*}{ DIMENSIONES } & \multicolumn{2}{|c|}{$\begin{array}{l}\text { Estadísticos descriptivos de } \\
\text { la diferencia }\end{array}$} & \multirow{2}{*}{ T Student } & \multirow{2}{*}{$\begin{array}{l}\text { Grados } \\
\text { de } \\
\text { libertad }\end{array}$} & \multirow{2}{*}{ P valor } & \multirow{2}{*}{ d Cohen } & \multirow{2}{*}{$\mathrm{r}$} \\
\hline & Media & $\begin{array}{l}\text { Desviación } \\
\text { estándar }\end{array}$ & & & & & \\
\hline IMPACTO & 4,78 & 9,24 & 9,380 & 328 & $<0,01$ & 0,7 & 0,3 \\
\hline INTERPERSONAL & 6,42 & 4,36 & 26,685 & 328 & $<0,01$ & 1,89 & 0,6 \\
\hline $\begin{array}{l}\text { COMPETENCIAS Y } \\
\text { EXPECTATIVAS }\end{array}$ & 1,83 & 4,35 & 7,664 & 328 & $<0,01$ & 0,3 & 0,1 \\
\hline TOTAL & 13,04 & 14,38 & 16,453 & 328 & $<0,01$ & 1,12 & 0,48 \\
\hline
\end{tabular}

Fuente. Elaboración propia a partir de los datos de estudio de 2016.

El gráfico 2 representa el cambio de la variable sobrecarga del cuidador en la pre y pos prueba del estudio.

Gráfico 2. Sobrecarga del cuidador pre y pos prueba.

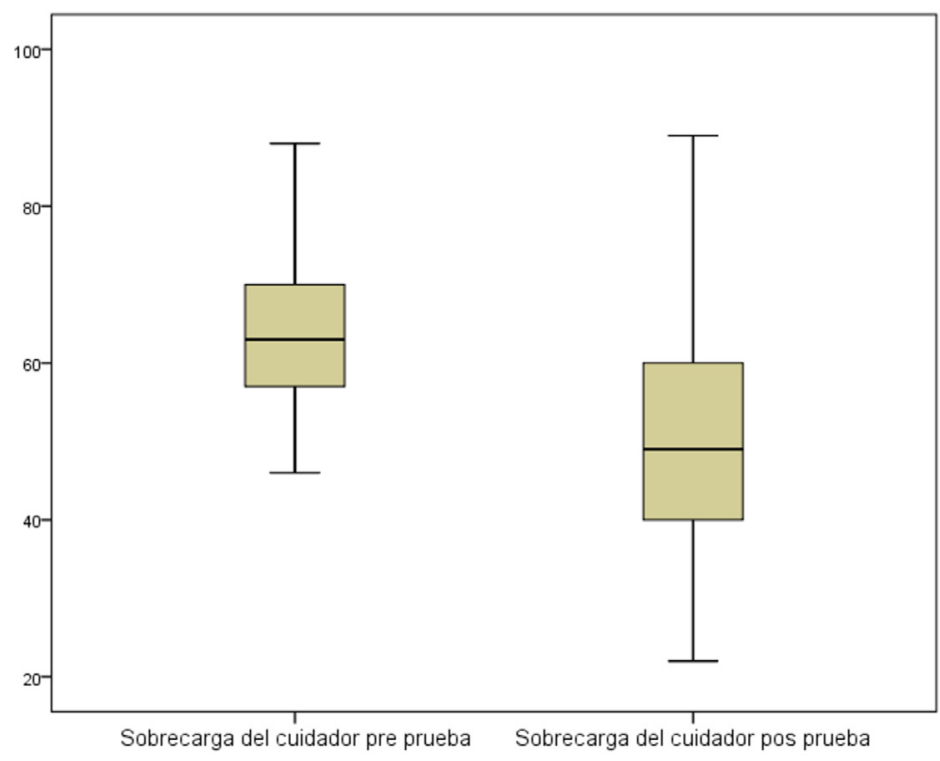

Fuente. Elaboración propia a partir de los datos de estudio de 2016. 


\section{Discusión}

Dentro de las características de las personas con ECNT es llamativo que el 47,5 \% presentan algún grado de dependencia y que un $29,5 \%$ presentan deterioro cognitivo, variables que se relacionan con la sobrecarga del cuidador y sustentan el requerimiento del cuidado continuo (19). Además, se evidencia que, en un $66,6 \%$, estas personas sienten que son una carga para sus familias, situación que merece ser estudiada.

En cuanto a los cuidadores familiares, se observa un perfil típico con predominancia femenina, edad media de 46,9 ańos, niveles intermedios de escolaridad, estrato socioeconómico entre 1 y 3 , con mayor ocupación al hogar, en su mayoría únicos cuidadores, con una media de 137,1 meses de dedicación al cuidado. Lo anterior es relevante considerando que, el ser mujer, con una alta dedicación al cuidado, la falta de recursos económicos y la baja escolaridad son aspectos que se relacionan con la habilidad para cuidar y la sobrecarga del cuidador (20-27).

El programa "Cuidando a los Cuidadores", en su versión institucional, tiene un efecto en el aumento de la habilidad de cuidado $(\mathrm{p}<0.01)$ y sus dimensiones conocimiento, valor y paciencia $(\mathrm{p}<0.01)$. Se estimó un tamaño de efecto significativo con un coeficiente d de cohen cercano a una desviación estándar en la variable total y por dimensiones, además un coeficiente $r$ entre 0.37 y 0.43 , lo cual muestra un efecto moderado de la intervención en las variables y sus dimensiones. Estos hallazgos son coherentes con lo reportado en los estudios de Díaz y Rojas (4), Barrera y otros (10), Vega y otros (11), excepto en la dimensión valor, la cual no había presentado cambio en los estudios mencionados. Un hallazgo novedoso en el presente estudio es el cálculo de tamaño de efecto, indicador que hasta el momento no había sido reportado por ningún autor y del cual no se tienen referentes de comparación (9-11).

Además del efecto sobre la habilidad de cuidado, el programa tuvo un efecto en la disminución de la sobrecarga de cuidador $(\mathrm{p}<0.01)$ y en sus dimensiones de impacto, interpersonal, competencias y expectativas $(\mathrm{p}<0.01)$. Además, mostró un tamaño de efecto importante con un coeficiente d de Cohen de 1,12 desviaciones estándar y $\mathrm{r}$ de 0,48 en la variable total. En las dimensiones impacto e interpersonal presentó un tamańo de efecto moderado con una d de Cohen entre 0,7 y 1,89 desviaciones estándar y $\mathrm{r}$ entre 0,3 y 0,6. Mientras que en la dimensión competencias y expectativas tuvo un tamaño de efecto débil con una d de Cohen de 0,3 desviaciones estándar y $\mathrm{r}$ de 0,1 . Estos hallazgos aportan a la prueba del supuesto del modelo para abordar la carga del cuidado de la ECNT, que postula una relación inversa entre la habilidad de cuidado y la sobrecarga del cuidador. Se puede decir que el componente interpersonal tiene mucha relación con otra variable que es el soporte social y familiar y se ha encontrado que disminuye la ansiedad y la posibilidad de angustia y estrés en el cuidador (28-31).

Es una limitación de este estudio el haberse conducido sin un grupo control, por lo que se sugiere hacerlo en próximas pruebas del programa. Como se realizó una única medición pos prueba de forma inmediata al finalizar la intervención, para posteriores estudios se sugiere hacer seguimiento al efecto a través del tiempo con medidas repetidas de las variables de resultado.

\section{Conclusiones}

El programa "Cuidando a los Cuidadores", versión institucional, tiene efecto en el aumento de la habilidad de cuidado $(\mathrm{p}<0,01)$ y disminución de sobrecarga del cuidador $(\mathrm{p}<0,01)$, con un tamaño de efecto moderado para ambas variables. El programa es aplicable en la práctica y replicable en la investigación. Se requiere probar el programa en diseńos con dos grupos y con medidas repetidas de las variables.

Desde lo conceptual disciplinar de enfermería, el programa es un indicador empírico novedoso y 
válido para probar que la carga del cuidado es un fenómeno medible y modificable positivamente en la experiencia de ser cuidador y recibir un cuidado de calidad.

Algunos supuestos del modelo para abordar la carga del cuidado en la enfermedad crónica están siendo validados con esta investigación, lo que permite avanzar en el conocimiento propio hacia la definición de teoría de rango medio derivada de investigación y producida en América Latina, que cuenta con todos los elementos conceptuales, teóricos y empíricos que requiere la comprobación de la ciencia de enfermería.

\section{Referencias}

1. Barrera L, Pinto N, Sánchez B, Carrillo G, Chaparro L. Cuidando a los cuidadores familiares de personas con enfermedad crónica. Bogotá: Editorial Universidad Nacional de Colombia; 2010 .

2. Kleinman A. Caregiving as moral experience. The Lancet. 2012; 380(9853): 1550-1551

3. Given B, Wyatt G, Given C, Gift A, Sherwood P, DeVoss $\mathrm{D}$, Rahbar M. Burden and depression among caregivers of patients with cancer at the end-of-life. Oncol Nurs Forum. 2004; 31(6): 1105-1117.

4. Barrera L, Galvis CR, Moreno ME, Pinto N, Pinzón ML, Romero E, Sánchez B. La habilidad de cuidado de los cuidadores familiares de personas con enfermedad crónica: un estudio comparativo de tres unidades académicas de enfermería. Invest Educ Enferm. 2006; 24(1): 36-46.

5. Carrillo GM, Barrera L, Sánchez B, Carreño S, Chaparro L. Efecto del programa de habilidad de cuidado para cuidadores familiares de niños con cáncer. Rev. Colomb. Cancerol. 2014; 18(1): 18-26.

6. Barrera L, Sánchez B, Pinto N. "Cuidando a los cuidadores". Un programa de apoyo a familiares de personas con enfermedad crónica. Index Enferm. 2006; 15(52): 54-58.

7. Nkongo N. The Caring Ability Inventory. In: Strickland O, Dilorio C, editors. Measurement of Nursing Outcomes Self Care and Coping. New York: Springer Publishing Company; 2003. 181-195 p.

8. Montalvo A, Flórez I, Stavro D. Cuidando a cuidadores familiares de niños en situación de discapacidad. Aquichan. 2008; 8(2): 197-211.

9. Barrera L, Pinto N, Sánchez B. Evaluación de un programa para fortalecer a los cuidadores familiares de enfermos crónicos. Rev. Salud pública. 2006; 8 (2):141-152.
10. Díaz J, Rojas MV. Cuidando al cuidador: efectos de un programa educativo. Aquichan. 2009; 9(1): 73-92.

11. Vega OM, Mendoza M, Ureña M, Villamil WA. Efecto de un programa educativo en la habilidad de cuidado de los cuidadores familiares de personas en situación crónica de enfermedad. Rev. Cienc. Cuidado. 2008; 5(1): 5-19.

12. Barrera L, Carrillo GM, Chaparro L, Sánchez B. Modelo para abordar la carga del cuidado de la enfermedad crónica en Colombia. Orinoquia. 2015; 19(1): 89-99.

13. García BVS. Manual de métodos de investigación para las ciencias sociales: un enfoque de enseńanza basado en proyectos. México: Manual Moderno; 2009.

14. Hernández R, Fernández C, Baptista P. Metodología de la investigación. Mexico: McGraw-Hill/Interamericana; 2006.

15. Chaparro L, Sánchez B, Carrillo GM. Encuesta de caracterización del cuidado de la diada cuidador familiar - persona con enfermedad crónica. Rev. Cienc. Cuidado. 2014; 11(2): $31-45$.

16. Barrera L, Sánchez B, Carrillo GM, Chaparro L, Carreño SP. Validación semántica y prueba de estabilidad del "Inventario de habilidad de cuidado" versión en español Actual Enferm. 2015; 18(1): 8-14.

17. Barreto R, Campos MS, Carrillo GM., Coral R, Chaparro L. Duran M y otros. Entrevista Percepción de Carga del Cuidado de Zarit: pruebas psicométricas para Colombia. Aquichan. 2015;15(3), 368-380.

18. República de Colombia. Ley 911 de 2004. Por la cual se dictan disposiciones en materia de responsabilidad deontológica para el ejercicio de la profesión de Enfermería en Colombia. Bogotá, Colombia: Congreso de la Republica de Colombia; 2004.

19. Akpan-Idiok PA, Anarado AN. Perceptions of burden of caregiving by informal caregivers of cancer patients attending University of Calabar Teaching Hospital, Calabar, Nigeria. Pan Afr Med J. 2014; 18:159.

20. Abellán M J, López C, Santi MJ, Deudero M, Picardo, J. M. ¿Quién es el receptor de intervenciones sociosanitarias y cuáles son sus necesidades? Enferm Clínica. 2016; 26(1): 49-54.

21. Arias M, Barrera L, Carrillo G, Chaparro L, Sánchez B, Vargas E. Cuidadores familiares de personas con enfermedad crónica en las regiones de frontera colombiana: perfil y carga percibida de cuidado. Rev Fac Med. 2014; 62(3): 387-397.

22. D’Onofrio G, Sancarlo D, Addante F, Ciccone F, Cascavilla L, Paris, F and others. Caregiver burden characterization in patients with Alzheimer's disease or vascular dementia. Int J Geriatr Psychiatry. 2015; 30(9): 891-899.

23. González F, Graz A, Pitiot D, Podestá J. Sobrecarga del cuidador de personas con lesiones neurológicas. Rev del Hospital JM Ramos Mejía. 2004; 9(4): 1-9.

24. Montalvo A, Flórez I. Características de los cuidadores de personas en situación de cronicidad. Cartagena (Colombia) Un estudio comparativo. Salud Uninorte. 2008; 24(2): 181-190.

25. Naranjo Flórez Ricardo Andrés. Avances y perspectivas en Sín- 
drome de Asperger. Nova. 2014; 12 ( 21 ): 81-101.

26. Almonacid Urrego Carmen Cecilia, Camarillo Romero María del Socorro, Gil Murcia Zulay, Medina Medina Claudia Yasmin, Rebellón Marulanda Jennifer Viviana, Mendieta Zerón Hugo. Evaluación de factores de riesgo asociados a enfermedad cardiovascular en jóvenes universitarios de la Localidad Santafé en Bogotá, Colombia. Nova. 2016; 14(25): 9-17.

27. González Devia Johanna L., Monroy Romero Paola A., Almonacid Urrego Carmen C.. Homocisteína y otros factores de riesgo cardiovascular en niños de educación básica primaria del Colegio Distrital Manuel Elkin Patarroyo, Bogotá, D.C. Colombia. Estudio piloto. Nova. 2017 ; 15( 27 ): 103-117.

28. Rosell-Murphy M, Bonet-Simó JM, Baena E, Prieto G, Bellerino $\mathrm{E}$ and others. Intervention to improve social and family support for caregivers of dependent patients: ICIAS study protocol. BMC Fam Pract. 2014; 15(1): 53.

29. Bernal Luz Mery, López Greizy. Diagnóstico prénatal: retrospectiva. Nova. 2014; $12(21)$ ): 23-36.

30. Márquez Gómez Marco Antonio, Gómez Díaz Graciela María. Accidente ofídico en el departamento de Sucre, Colombia. Nova. 2015; 13( 24 ): 39-46.

31. González Yuri Lilian. Evaluación de la percepción del riesgo ocupacional en trabajadores de una empresa del sector de la construcción en Bogotá D.C. Nova. 2015; 13 ( 23 ): 93-107. 